\title{
TEMA: Patología anorectal frecuente en atención primaria
}
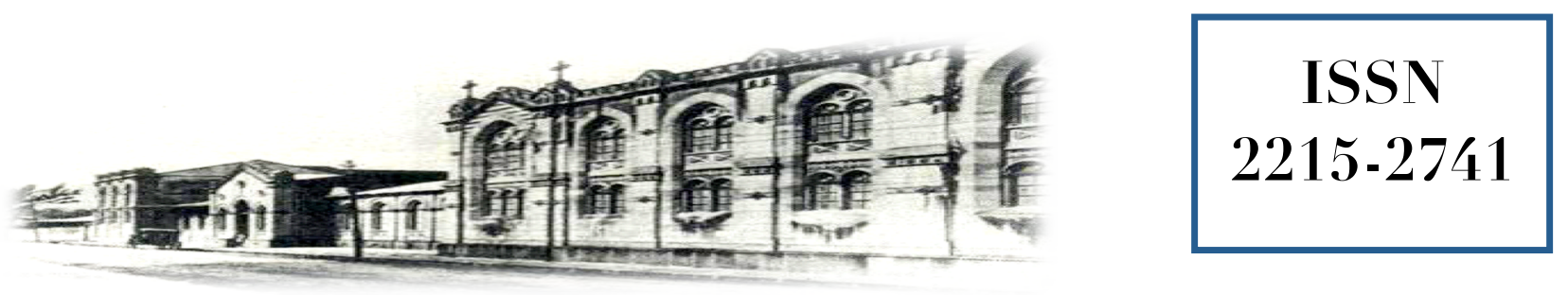

Hospital San quan de Dios. San Dosé. Costa Rica. Fundado en 1845

$\begin{array}{ll}\text { Recibido: } & 14 / 04 / 2016 \\ \text { Aceptado: } & 21 / 04 / 2016\end{array}$

Aceptado: $\quad 21 / 04 / 2016$

Andrés Leitón Chaves ${ }^{1}$

Victoria Vargas López ${ }^{2}$

${ }^{1}$ Médico y Cirujano. Profesor Universidad Autónoma de Centroamérica, Escuela de Medicina. andres131c_@hotmail.com

${ }^{2}$ Médico y Cirujano. Especialista en Cirugía General, Hospital San Rafael de Alajuela. Victo_vl@yahoo.com

\section{RESUMEN}

Las enfermedades relacionadas al anorecto son comunes de observar en el primer nivel de atención, es generalmente el consultorio del médico general donde el paciente consultara primariamente sobre su patología. Es importante el conocimiento por parte de los médicos de atención primaria acerca de los aspectos básicos de las consultas más frecuentes en proctología, las entidades clínicas incluyendo su etiología, fisiopatología y tratamiento, pero principalmente su diagnóstico clínico así como el reconocimiento del momento en que es necesario referir a un paciente con un padecimiento anorectal a un nivel más especializado. El siguiente artículo consta de una revisión de la evidencia actual sobre los aspectos a considerar en el diagnóstico y manejo de la patología proctológica, esto con el objetivo de optimizar la atención brindada en los primeros niveles de salud.

\section{PALABRAS CLAVE}

Anorectal. Proctología. Atención primaria. Fisura. Fístula. Hemorroides. Incontinencia.

\begin{abstract}
Anorectum diseases are common to observe in the first level of care, is usually the general practitioner's office where the patient consulted primarily about their condition. It is important to know by primary care physicians about the basics of the most common queries in proctology and clinical entities, including its etiology, pathophysiology and treatment, but mainly clinical diagnosis and recognition of time it is necessary to refer a patient with anorectal at a more specialized level condition. The following article contains a review of the current evidence on the aspects to be considered in the diagnosis and management of proctologic disease, this in order to optimize the care provided in the first levels of health.
\end{abstract}




\section{KEY WORDS}

Anorectal. Proctology. First care. Fissure. Fistula. Hemorrhoids. Incontinence.

\section{HISTORIA DE LA PROCTOLOGIA}

Medicina antigua Egipcia: En el estudio de la medicina Egipcia antigua, lo más importante eran los antiguos papiros médicos escritos entre los años 3000 y 1200 A.C. En el papiro de Eber's se mencionan diversos revestimientos y grasas, tales como manteca de cerdo con hojas de acacia contra el dolor de las hemorroides. Por otra parte hasta la era Romana tardía, circuló una leyenda de que los primeros médicos egipcios descubren e introducen por primera vez en la práctica médica uno de los procedimientos terapéuticos más realizados contra la acumulación de heces en el tracto digestivo inferior (enema), esto lo aprendieron del pájaro Ibis (Dios Toth), un residente permanente de las zonas costeras del Río Nilo. Los egipcios creían que permaneciendo de pie en el Nilo, el pájaro Ibis introducía agua en el recto con su largo pico lavando la parte final del colon.

Medicina India antigua: Uno de los más importantes escritores médicos en la India antigua fue Sushruta Samhita quién habló sobre las fístulas perianales y su tratamiento quemándolas con hierro caliente (cauterización) y tirando de las fibras impregnadas con alcaloides vegetales que destruyen el tejido del callo de la fístula y fomentan el crecimiento fresco del tejido de granulación (terapia Kshrasutra).

Medicina griega Hipocrática antigua: Las cirugías para fístulas fueron llevadas a cabo por médicos de la escuela Hipocrática tirando de hilos de lino a través de la fístula, utilizando una sonda de estaño, tallándola gradualmente conduciendo al corte de la fístula.

Medicina Romana antigua: Celsus, entre otras cosas, describió la operación de las hemorroides mediante ligadura y la cirugía de la fístula de dos maneras: ligadura e incisión. El mayor médico romano y uno de los médicos más grandes de todos los tiempos, Galeno, fue el primero en describir anatómicamente los músculos del ano.
También fue conocido como el inventor del "Syringotom" para la incisión de la fístula.

Medicina Árabe: El más famoso médico árabe y uno de los más grandes médicos de todos los tiempos, Avicena, en su primer libro "Canon", describió la anatomía del esfínter anal y explicó su procedimiento en el tratamiento quirúrgico de las fístulas perianales usando hilo de seda para su ligadura.

Medicina del Renacimiento: Andreas Vesalius, en su monumental trabajo "De humani corporis fabrica", primero describe la técnica de disección de la parte final del colon. Además, describió el esfínter anal externo y dos músculos, elevadores del ano. Girolamo Fabrizi ab Acquapendente, en su libro "Opera chirurgica" describe las fístulas anales y su tratamiento, algunos lo consideran el gran "proctólogo del renacimiento".

Desarrollo de la proctología en la medicina moderna: Un repentino progreso en el diagnóstico y tratamiento de enfermedades anorrectales se produjo después de la Segunda Guerra Mundial y continuó hasta nuestros días. Mejores condiciones, rectoscopios más perfectos $\mathrm{y}$ un rápido progreso en todos los campos de la medicina, especialmente la cirugía, causaron un interés cada vez mayor en los cirujanos de hoy en día en la patología anorrectal ${ }^{1}$.

\section{HISTORIA CLÍNICA Y EXAMEN FÍSICO EN PATOLOGÍA ANORECTAL}

La exploración de la patología ano-rectal debe transcurrir en un ambiente sereno y tranquilo, preservando la intimidad necesaria, sin ruidos ni interrupciones, que genera confianza en el paciente a fin de superar la incomodidad del momento. Debe explicarse al paciente que se le va a hacer y que puede sentir durante la misma, a fin de evitar situaciones violentas. 


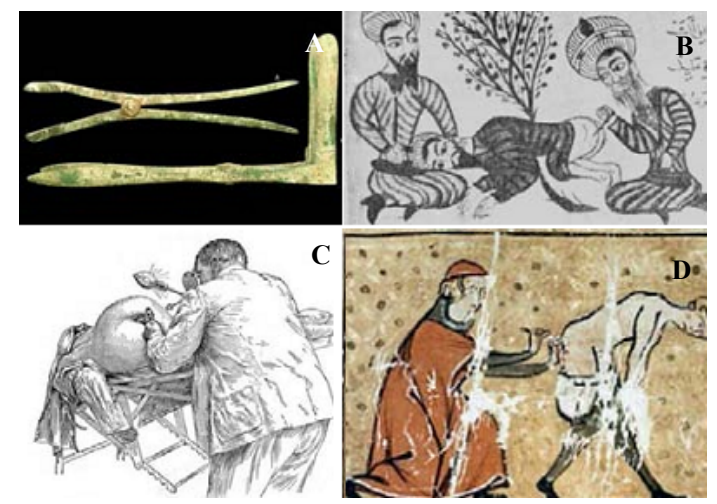

Figura 1. A. antiguo espéculo rectal griego y romano, B. del libro Sharaf ed Din-anorectal examine, C. examen rectoscópico con espéculo Kelly, D. examen anorectal-edad media

Fuente: www.onk.ns.ac.rs/Archive Vol 21, No. 1, March 2013

\section{Historia clínica}

$\checkmark$ Antecedentes familiares oncológicos: (Incidencia de cáncer colorrectal en familiares de primer y segundo grado).

$\checkmark \quad$ Antecedentes personales: enfermedades y tratamientos médicos habituales pueden influir de algún modo en los síntomas del proceso anorrectal como patología tiroidea, patología hepática, enfermedad inflamatoria intestinal, tratamiento antiagregante o anticoagulante, enfermedades neurológicas, tratamiento de patología psiquiátrica.

$\checkmark$ Cirugía anorectal previa: puede condicionar el desarrollo de lesiones cutáneas perianales y tono del ano.

$\checkmark$ Prácticas sexuales: Pueden condicionar el desarrollo de lesiones cutáneas perianales (herpes, VPH) o en el canal anal ${ }^{2}$

\section{EXPLORACIÓN FÍSICA EN PROCTOLOGÍA}

Se recomienda explorar al paciente en posición de Sims, decúbito lateral izquierdo con las piernas flexionadas sobre el abdomen, en diagonal sobre la camilla, con el hombro derecho ligeramente inclinado hacia el lado izquierdo, favoreciendo de este modo la exposición anal y perianal.

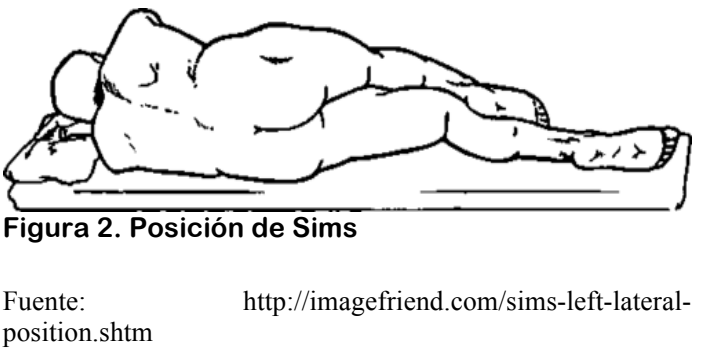

La localización de la patología anal debe describirse según su ubicación anatómica (anterior, posterior, lateral izquierdo, lateral derecho) evitando la nomenclatura horaria.

1. Inspección anal: Se realiza una separación cuidadosa de ambas nalgas, se podrá así valorar la presencia de secreciones, colgaos cutáneos o "tags" perianales, hidrosadenitis o foliculitis perianal, hemorroides externas, fisuras, fístulas, prolapso mucoso, prolapso rectal, tumoraciones o ulceraciones. Se le puede pedir al paciente que realice una maniobra defecatoria suave lo cual pondrá de manifiesto lesiones que pueden pasar desapercibidas en reposo, pues de este modo se acentúan los prolapsos de lesiones del canal anal o de la pared rectal.

2. Palpación perianal: Identificar puntos dolorosos, induraciones, posibles trayectos fistulosos, masas.

3. Tacto rectal: permite identificar puntos dolorosos, masas, abscesos y comprobar el tono esfinteriano. Debe realizarse con cuidado, lubricando el dedo índice enguantado, se presiona suavemente el orificio anal antes de introducirlo para vencer la presión de reposo. Una vez introducido el dedo se llega a la altura máxima para, en retirada, ir palpando toda la circunferencia ano-rectal. Se valora si existe alguna lesión intraluminal, o tumoraciones. Se palpa la próstata en la parte anterior del recto, posteriormente el canal anal, y finalmente tras su retirada se inspecciona el guante para verificar la presencia de manchado patológico.

4. Anoscopía: con un anoscopio tubular (7 $\mathrm{cm}$ de longitud) se explora la

mucosa del recto distal, el plexo hemorroidal interno, línea

pectínea y anodermo en busca de lesiones mucosas, crecimiento endoluminal, orificios fistulosos y fisuras. Durante el retiro se observa al pujar si se pone de manifiesto 
una procidencia oculta.

En el estudio de Caneesa C, "Examen proctológico y tacto rectal en el diagnóstico clínico de síntomas anorrectales" concluyen que un examen proctológico completo permite establecer un diagnóstico clínico primario en la mayoría de los pacientes que consultan por síntomas anorrectales. Más de la mitad de las condiciones patológicas del anorecto pueden ser puestas en evidencia mediante una correcta inspección y palpación anoperineal; si bien la anoscopía incrementa el rendimiento diagnóstico, solo suele estar disponible en consultorios especializados $^{2}$.

\section{ANATOMÍA DEL CANAL ANAL}

El recto: Se extiende desde el nivel del promontorio sacro al nivel del elevador del ano, y tiene aproximadamente $15 \mathrm{~cm}$ de longitud. Difiere del colon en que, la capa longitudinal muscular es continua, lo opuesto a la condensación en tres tenias en el colon. Tiene dos o tres curvaturas que forman pliegues submucosos en el lumen conocidos como válvulas de Houston.

Canal anal y esfínteres: El canal anal, de $4 \mathrm{~cm}$ de longitud, se extiende desde el recto, a medida que pasa a través del elevador del ano, cruza el borde anal y se extiende a la piel del ano. . La pared muscular del canal anal forma el esfínter anal interno. En canal anal está envuelto inferiormente por el esfínter anal externo, que se origina del músculo puborectal. A $2 \mathrm{~cm}$ del borde anal se encuentra la línea pectínea.

Los pliegues longitudinales por arriba de la línea pectínea se conocen como columnas de Morgagni. A $1 \mathrm{~cm}$ por encima de la línea pectínea está la zona de transición de epitelio columnar a escamoso estratificado. El canal anal quirúrgico se extiende desde el anillo anorectal al borde anal. El anodermo describe la piel del borde anal: piel sin vellos distal a la línea dentada, pero proximal a la piel vellosa del margen anal. La piel perianal (piel vellosa) se extiende del borde lateral del margen anal a 5-6 $\mathrm{cm}$ del margen anal.

Irrigación del recto y canal anal: $\mathrm{La}$ arteria hemorroidea superior es continuación de la AMI, la arteria hemorroidea media se origina de las iliacas internas, la hemorroidea inferior se origina de la arteria pudenda interna, irriga los esfínteres anales.
Drenaje linfático: El recto superior y medio drena a ganglios mesentéricos, el recto inferior drena a los nodos mesentéricos inferiores pero también a nódulos iliacos internos. El drenaje linfático de la piel perianal es completamente hacia nódulos inguinales.

Inervación del recto y el canal anal: Las fibras simpaticas del recto provienen de los tres primeros segmentos lumbares. La inervación parasimpática pélvica proviene de nervios esplácnicos pélvicos (S2-S3-S4). Ambos simpatico y parasimpático inervan el EAI, que mantiene la contracción tónica. El EAE es inervado por la rama rectal inferior del nervio pudendo. La sensibilidad del canal anal viene del nervio rectal inferior. Las sensaciones dolorosas del canal anal pueden sentirse a $1.5 \mathrm{~cm}$ proximales a la línea dentada. Hay falta de sensación somática por encima de la línea dentada ${ }^{3}$.

Síntomas habituales en patología anorectal

$\checkmark$ Rectorragia: emisión de sangre roja por el ano. También se denomina hematoquecia y debe distinguirse de la melena, deposiciones negras y pastosas de sangre ya digerida. La rectorragia puede ser aguda, masiva o moderada o bien crónica, de pequeña cuantía pero mantenida en el tiempo.

$\checkmark$ Dolor: La proctalgia es un dato muy prevalente, es importante conocer su duración e intensidad, los factores desencadenantes y si se asocia con emisión de sangre o pus, coexistencia con alteración del tránsito intestinal (estreñimiento/diarrea), fiebre, tumoraciones anales/perianales.

$\checkmark$ Emisión de secreciones por ano: secreción acuosa (adenoma velloso rectal), mucosa (dicha secreción es habitual en el colon pero puede aumentar en caso de tumores, EII, intestino irritable o por exteriorización de la mucosa anorectal en el caso de prolapsos mucosos o rectales), secreción purulenta (secundaria a procesos infecciosos como la gonorrea o proctitis por Chlamydia trachomatis, o procesos infecciosos bacterianos que afectan a tramos distales del colon) o escapes/ensuciamientos de heces en pacientes con incontinencia anal. 
Tabla 1. Hallazgos clínicos y diagnósticos sugestivos.

\begin{tabular}{|c|c|c|}
\hline $\begin{array}{l}\text { Tipo de } \\
\text { dolor }\end{array}$ & Hallazgo & $\begin{array}{l}\text { Sospecha } \\
\text { diagnóstica }\end{array}$ \\
\hline \multirow{5}{*}{ Continuo } & $\begin{array}{l}\text { Tumefacción } \\
\text { perianal más } \\
\text { fluctuación. }\end{array}$ & Absceso \\
\hline & Nódulos anales. & Hemorroides \\
\hline & $\begin{array}{l}\text { Inspección } \\
\text { normal con } \\
\text { fiebre. }\end{array}$ & $\begin{array}{c}\text { Absceso } \\
\text { submucoso, } \\
\text { criptitis, } \\
\text { prostatis aguda. }\end{array}$ \\
\hline & $\begin{array}{l}\text { Inspección } \\
\text { normal, sin } \\
\text { fiebre y con } \\
\text { alteraciones del } \\
\text { ritmo. }\end{array}$ & $\begin{array}{l}\text { Sospecha de } \\
\text { neo de recto. }\end{array}$ \\
\hline & $\begin{array}{l}\text { Inspección } \\
\text { normal, con } \\
\text { estreñimiento y } \\
\text { en ancianos. }\end{array}$ & Fecaloma \\
\hline Discontinuo & $\begin{array}{l}\text { Hemorragia } \\
\text { postdefecación }\end{array}$ & Fisura anal \\
\hline
\end{tabular}

Tumoraciones o masas: podemos identificar tumoraciones perianales dolorosas (abscesos, hidrosadenitis perianal), o bien induraciones en área sacrococcígea (sinus pilonidal), y tumoraciones anorectales propiamente, que pueden ser reductibles (hemorroides grado III, pólipos rectales, papila anal prolapsada, tumores rectales, prolapso rectal) o irreductibles (colgajos cutáneos, hemorroides grado IV, trombosis hemorroidal, condilomas anales, neoplasias anales).

$\checkmark$ Alteración del ritmo intestinal: presencia de diarrea (aumento de volumen de las heces, menor consistencia, y mayor frecuencia deposicional) o estreñimiento (defecación dificultosa, de escasa frecuencia y acompañada de asociación de evacuación incompleta).

Tenesmo rectal: necesidad persistente de hacer deposición, incluso tras una defecación en principio satisfactoria. Se debe habitualmente a la ocupación de la ampolla rectal (fecaloma, neoplasia). $\checkmark$ Prolapso: Podemos encontrar prolapso de elementos del canal anal y el recto (tumoraciones, pólipos, hemorroides grado III/IV) o bien propiamente el descenso circunferencial de la pared rectal, que puede ser completo o parcial.

$\checkmark \quad$ Incontinencia: Incapacidad para el control voluntario de la emisión de heces y gases, incluye también afectación de la capacidad de discriminar entre heces y gases. Se clasifica como incontinencia grado 1 cuando existe limitación de incontinencia a gases, grado 2 cuando afecta la continencia de heces líquidas, y grado 3 cuando hay incapacidad de contener heces sólidas.

$\checkmark \quad$ Urgencia defecatoria: Necesidad imperiosa de hacer defecación.

$\checkmark$ Prurito: No es una enfermedad en sí, sino un síntoma. Puede ser agudo o crónico y, según su etiología, secundario o idiopático. Se puede encontrar como patología subyacente un eccema perianal, psoriasis, dermatitis de contacto, asociarse a exposición a sustancias irritantes locales o bien deberse a la irritación secundaria por la secreción mucosa en el prolapso rectal ${ }^{2}$.

\section{SÍNDROMES DE PROCTALGIA}

La proctalgia crónica se define como episodios recurrentes de dolor rectal, con una duración de cada episodio de 20 minutos o más. Es también llamada síndrome del elevador del ano, espasmo del elevador, síndrome puborectal, síndrome piriforme $\mathrm{y}$ mialgia por tensión pélvica. Todos los tratamientos comúnmente usados para la proctalgia crónica son dirigidos directamente a la relajación de los músculos del piso pélvico, asumiendo que la proctalgia crónica es debida a una contracción sostenida de los músculos del piso pélvico. Evidencia reciente sugiere que la fisiopatología de la proctalgia crónica y la defecación por disinergia pueden solaparse.

Recomendaciones para el diagnóstico: El diagnóstico de proctalgia crónica debe 
hacerse basado en la historia de episodios recurrentes de dolor rectal, cada uno de al menos 20 minutos de duración, un examen rectal que demuestre sensibilidad a la palpación del elevador del ano y exclusión de otras causas de dolor rectal. Los gastroenterólogos y otros especialistas deben obtener estudios de imagen o endoscópicos para descartar causas estructurales de dolor rectal. En un estudio grande de tratamiento, el fallo para evacuar un balón lleno con $50 \mathrm{ml}$ de agua y la inhabilidad para relajar los músculos del piso pélvico se correlacionaron con la presencia de sensibilidad a la palpación y fueron predictivos para el éxito de la terapia de retroalimentación (biofeedback). Estas observaciones sugieren que el test de expulsión del balón y la manometría anorectal deben añadirse al proceso de diagnóstico para mejorar la selección de pacientes para tratamiento de retroalimentación.

Recomendaciones para el tratamiento: El tratamiento preferido es la terapia de retroalimentación para enseñar a relajar el piso pélvico. La estimulación eléctrica es superior al masaje digital pero inferior a la retroalimentación.

La proctalgia fugaz, se caracteriza por sensaciones intensas de dolor rectal o del canal anal con una duración de apenas pocos segundos, debe distinguirse de la proctalgia crónica, en la que los episodios de dolor son más prolongados. La fisiopatología de la proctalgia fugaz se desconoce, aunque se ha reportado engrosamiento del esfínter anal interno y presión de reposo elevada en el canal anal, estos hallazgos sugieren espasmo del esfínter anal interno.

Recomendaciones para el diagnóstico: El dianóstico de proctalgia fugaz debe basarse en la historia de ataques intermitentes de dolor severo en el canal anal o el recto inferior con una duración menor a 20 minutos. Se deben descartar causas estructurales de dolor anorectal (fisura anal, hemorroides, criptitis, malignidad) mediante estudios de imagen, endoscópicos $\mathrm{u}$ otros.

Recomendaciones para el tratamiento: Se debe explicar al paciente que la patología es benigna, la evidencia en tratamientos específicos es sólo anecdótica. Jeyarajah et al recomiendan la siguiente progresión de pasos para el paciente con episodios frecuentes y debilitantes de proctalgia fugaz:

1. Baños de agua caliente.

2. Trinitato de glicerilo tópico al $0.2 \%$ P.R.N

3. Inhalación de salbutamol 200g P.R.N

4. Enemas de agua caliente

5. Clonidina 150g B.I.D

6. Bloqueo anestésico local

7. Inyección de toxina botulínica en los esfínteres anales.

Para pacientes con engrosamiento demostrado del esfínter anal interno $\mathrm{y}$ presiones aumentadas del canal anal, estos autores consideran que una esfinterectomía lateral interna debe considerarse. La guía del manejo de desordenes anorectales benignos del colegio americano de gastroenterólogos no avala estas recomendaciones por que la evidencia de estos tratamientos es sólo anecdótica, y algunos de estos manejos pueden asociarse a efectos adversos. El paciente típico con proctalgia fugaz tiene episodios infrecuentes y cortos de dolor para los que ni el tratamiento ni la prevención son prácticos $^{4}$.

\section{INCONTINENCIA FECAL}

La incontinencia fecal es la pérdida involuntaria de heces sólidas o líquidas. El término incontinencia anal, también incluye la pérdida involuntaria de flatos. La prevalencia de incontinencia fecal aumenta con la edad, más del $50 \%$ de los residentes en un hogar de cuido tenían incontinencia fecal, según una encuesta.

Etiología y factores de riesgo: En estudios epidemiológicos la edad avanzada, aumento del índice de masa corporal, diarrea, urgencia rectal, colecistectomía, fistula anal, lesión anal no obstétrica, incontinencia urinaria, enfermedades crónicas (diabetes, EVC) y medicamentos psicoactivos están asociados a incontinencia fecal ${ }^{4}$.

Tabla 2. Causas comunes de incontinencia fecal

Debilidad del esfínter anal

Traumática: obstétrica, quirúrgica 
No traumática: esclerodermia, degeneración del EAI de etiología desconocida

Neuropatía: periférica o generalizada. Afecciones del piso pélvico: prolapso rectal, síndrome de perineo descendente. Condiciones inflamatorias: proctitis por radiación, enfermedad de Chron, colitis ulcerativa.

Desordenes del SNC: demencia, ECV, tumores cerebrales, esclerosis múltiple, lesiones de médula espinal.

Diarrea: síndrome de intestino irritable, diarrea post-colecistectomía.

Otras: retención fecal con desbordamiento, desordenes del comportamiento.

Fuente: ACG Clinical Guideline. Management of Benign Anorectal Disorders

\section{Clasificación de la incontinencia fecal:}

1. De urgencia: la pérdida de heces se produce a pesar de querer detener la defecación. Se debe generalmente a una disrupción esfinteriana.

2. Pasiva: la pérdida de gases y/o heces se produce sin tener conciencia de ello. Esto responde a una pérdida de percepción y/o mal funcionamiento de los reflejos rectoanales.

3. Solling o ensuciamiento: manchado de la ropa interior por pérdida de heces sin conocimiento. Ocurre por una evacuación incompleta o una alteración de la sensibilidad anorectal. También puede presentarse de forma transitoria después de cirugía anal. ${ }^{(2)}$

Recomendaciones para el diagnóstico: El diagnóstico puede resultar complicado debido a que la mayoría de las personas que lo padecen no lo consultan con su médico por vergüenza, para su diagnóstico es esencial su sospecha. El médico debe preguntar al paciente acerca de la presencia de IF directamente en lugar de depender de un reporte. Se deben buscar factores que predispongan a IF. La severidad de los síntomas debe determinarse cuantificando el tipo de heces utilizando la escala de Bristol, así como la frecuencia, cantidad de pérdida y la presencia de urgencia. $(2,4)$

Exploración física: incluye inspección, palpación de la región perianal y tacto rectal que descarte una tumoración anorectal como causa del ensuciamiento. La inspección revelará si existen cicatrices antiguas, la existencia de un ano entreabierto o la ausencia de un tabique anovaginal.

\section{Tratamiento:}

-Tratamiento conservador: Uno de los aspectos más importantes a tratar inicialmente en el manejo de la incontinencia fecal es la modificación del hábito intestinal reduciendo la frecuencia de las deposiciones y variando la consistencia de las heces. Las medidas educacionales incluyen una rutina horaria con el objetivo de estar libre posteriormente de un eventual episodio de incontinencia. Se recomienda dieta rica en fibra y suplementos de la misma para aumentar el volumen de las heces y tratar la diarrea suspendiendo los medicamentos y alimentos que puedan producirla.

-Tratamiento farmacológico: Enemas y supositorios de glicerina son útiles en incontinencia pasiva y ensuciamiento por evacuación incompleta para conseguir vaciar la ampolla rectal. Medicamentos antiflatulentos (dimeticona o lactasa, carbono activado). Medicamentos antidiarreicos (loperamida, codeína, colestiramina), antidepresivos tricíclicos (amitriptilina 20-25mg/día). Biofeedback (ejercicios de Kegel).

Atención especializada: Una vez instaurado el tratamiento y después de un seguimiento de 6-12 meses los pacientes que persisten con IF que limite su calidad de vida deben remitirse a consulta especializada.

- Estudios funcionales: ultrasonido endoanal y/o resonancia magnética, manometría anorectal, pruebas neurofisiológicas.

- Valoración de la severidad: se emplea una escala del grado de severidad de la incontinencia (escala de la Cleveland Clinic) y una escala que permita valorar el impacto de la IF sobre la calidad de vida del paciente (FIQL).

- Tratamiento: Según la causa hay diferentes alternativas. Tratamientos minimamente invasivos, como son el refuerzo en consulta de biofeedback, la inyección de aumentadores del canal 
anal y la neuromodulación de raíces sacras. Tratamientos invasivos como la reparación quirúrgica de los esfínteres (esfinteroplastía). Implante de esfínter artificial o la realización de un estoma definitivo que mejore la calidad de vida del paciente con incontinencia fecal. ${ }^{2}$

\section{ENFERMEDAD HEMORROIDAL}

Definición: Las hemorroides son estructuras fisiológicas constituidas por plexos vasculares arteriovenosos que forman un almohadillado a lo largo del canal anal. La enfermedad hemorroidal se define como una serie de síntomas y signos (dolor, prurito, prolapso, sangrado, etc.) atribuibles al tejido hemorroidal generalmente secundarios a alteraciones estructurales de éste (dilatación e ingurgitación) y/o de los tejidos de sostén.

\section{Clasificación y tratamiento:}

\section{Hemorroides internas}

Se sitúan proximalmente a la línea dentada y están cubiertas por mucosa rectal y mucosa de transición. Se subclasifican en cuatro grados dependiendo de la extensión del prolapso (tabla 3 ). Las rectorragias y el prolapso son los signos cardinales de la enfermedad hemorroidal interna. "La hemorragia" puede estar presente en todos los grados hemorroidales, y aparece generalmente en relación con la defecación. La sangre es roja y brillante, aparece tras la expulsión de heces limpias e impregna el papel al limpiarse. El "prolapso hemorroidal" es descrito por los pacientes como la exteriorización de la mucosa intestinal durante la defecación. En los grados III y IV, se asocian otros síntomas, como malestar perianal, prurito anal o manchado mucoso. La presencia de dolor indica, generalmente, la existencia de una trombosis hemorroidal y/o el atrapamiento en un ano hipertónico de los plexos hemorroidales. (5)

Diagnóstico: El diagnóstico de la enfermedad hemorroidal se basa en una historia clínica precisa y un examen clínico cuidadoso. La historia clínica debe incluir una descripción de los síntomas e información de los hábitos deposicionales del paciente. Hay que determinar el origen del sangrado, descartando otras patologías, siendo la más importante el cáncer de recto.
Tras la inspección se realizará un tacto rectal, que aunque no sirve para diagnosticar hemorroides, ayuda a realizar un diagnóstico diferencial con otras causas de rectorragia. Puede realizarse una anoscopía, esta prueba se puede llevar a cabo en la consulta de atención primaria. Se debe solicitar una colonoscopía completa ante cualquier síntoma infrecuente, antecedentes familiares de pólipos o cáncer colorrectal y edad superior a los 40 años $^{2}$.

Tabla3. Clasificación de las hemorroides internas

\begin{tabular}{c|l|}
\cline { 2 - 2 } Grado & \multicolumn{1}{|c|}{ Descripción } \\
\hline I & Protruyen con la defecación \\
\hline II & $\begin{array}{l}\text { Prolapso con la defecación, } \\
\text { desaparecen espontáneamente. }\end{array}$ \\
\hline III & $\begin{array}{l}\text { Requieren reducción manual posterior } \\
\text { al prolapso }\end{array}$ \\
\hline IV & Incapacidad de reducir el prolapso \\
\hline
\end{tabular}

Fuente: Evaluation and Management of Common Anorectal Conditions. American Family Physician. Volume 85, Number 6. March 15, 2012

Tratamiento: El objetivo del tratamiento de las hemorroides es la resolución de los síntomas. El tratamiento de las hemorroides sintomáticas debe ser inicialmente conservador y está orientado a facilitar el esfuerzo al defecar.

-Medidas higienico-dietéticas: dieta rica en fibra, ingesta abundante de agua, dieta exenta de especias, evitar el exceso de bebidas alcohólicas. Los baños de asiento se indican en episodios agudos, lo ideal es que utilice agua templada, más bien caliente, y sin permanecer mucho tiempo (no más de 10 minutos) para conseguir la relajación del esfínter anal.

-Tratamiento farmacológico: Las cremas y pomadas reducen el edema y la inflamación y no sirven para tratar la rectorragia o el prolapso, no deben utilizarse por más de 7-10 días. La mayoría contienen corticosteroides, anestésicos y antisépticos. Los anestésicos tópicos (mejoran las molestias y el prurito) no deben ser usados demasiado tiempo, porque producen hipersensibilidad y dermatitis de contacto; del mismo modo, el uso prolongado de corticosteroides puede provocar atrofia cutánea y sobreinfección.

Los medicamentos orales incluyen agentes formadores de masa fecal; los más utilizados 
son: salvado, psyllium, plantago ovata, semillas de lino y metilcelulosa. Los laxantes solo deben utilizarse si no se logra facilitar la deposición con las medidas mencionadas anteriormente. Los fármacos venotónicos han demostrado su utilidad en el tratamiento, sobre todo en fases iniciales o en las crisis agudas, especialmente cuando el sangrado es la manifestación principal. La administración de diosmina oral en pleno brote y a dosis elevadas $(300 \mathrm{mg} / 4$ veces al día) parece mejorar los síntomas.

-Tratamientos mínimamente invasivos. Ante el fracaso o la intolerancia a las medidas previas se puede optar por varios procedimientos que se realizan normalmente de forma ambulatoria. $\mathrm{La}$ ligadura con banda elástica es el procedimiento más utilizado, con éxito en el $70-80 \%$ de los pacientes y más eficaz que la escleroterapia. Por lo general, tras la aplicación de bandas elásticas, se produce una sensación de opresión leve en la zona anal y posteriormente aparece la lesión propia del desprendimiento de la hemorroide que ocurre sobre el quinto día. Ocasionalmente los pacientes pueden presentar dolor intenso, infecciones o trombosis de otras hemorroides. Existen métodos que utilizan láser, infrarrojos o coagulación bipolar para destruir las hemorroides internas cuya eficacia es similar a la escleroterapia e inferior a la ligadura.

-Cirugía: La cirugía de la enfermedad hemorroidal sólo es necesaria en el 5-10\% de los pacientes. La hemorroidectomía "abierta" es la técnica de elección en las hemorroides grado IV, en caso de asociarse a un prolapso mucoso importante se opta por una hemorroidectomía circular "cerrada".

2. Hemorroides externas: Se ubican distalmente a la línea pectínea y están recubiertas por el anodermo y la piel perianal.

Sospecha clínica: Se caracterizan por la aparición súbita de dolor anal continuo, intensificado con la defecación, que alcanza su máxima intensidad a los 2-3 días y desaparece en una semana. Ello es consecuencia de la trombosis del plexo hemorroidal.

Figura 2. Hemorroide externa trombosada.

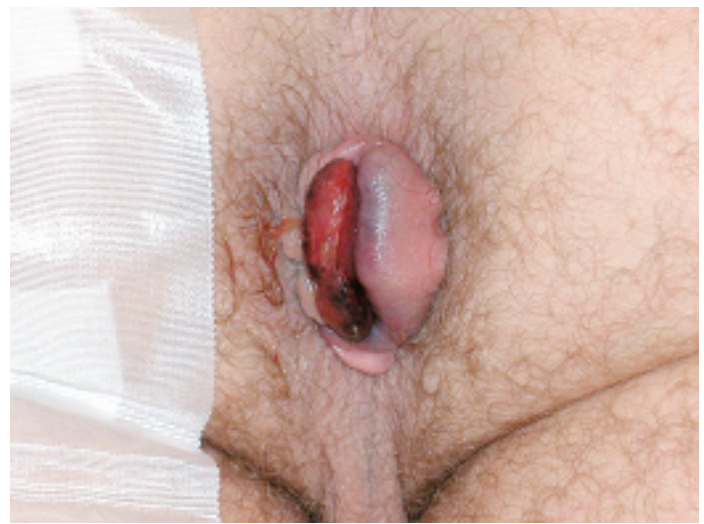

Fuente: Evaluation and Management of Common Anorectal Conditions. American Family Physician. Volume 85, Number 6. March 15, 2012.

Diagnóstico: En la inspección del ano se observa, en el margen anal, uno o varios nódulos subcutáneos, violáceos-azulados, dolorosos y duros al tacto, recubiertos de piel edematosa y en ocasiones ulcerada con salida parcial del trombo.

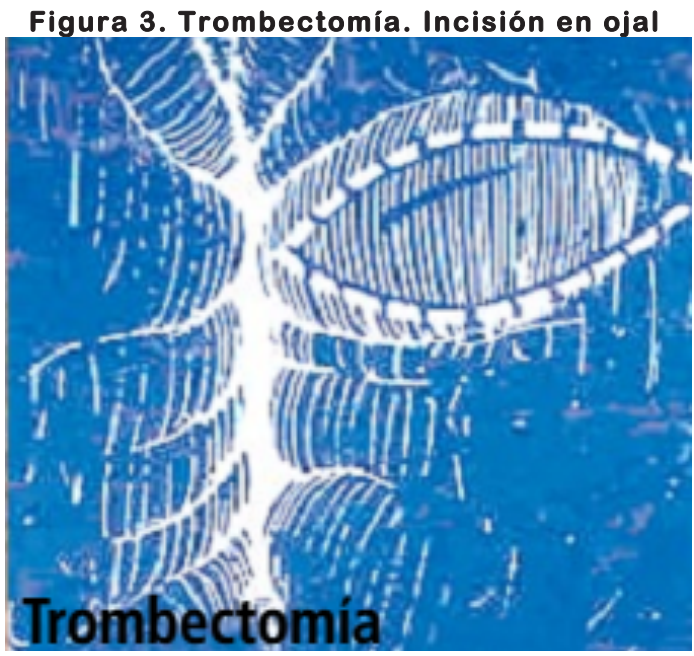

Fuente: REV. MED. CLIN. CONDES - 2011; 22(5) 677-684

Tratamiento: Además de las medidas conservadoras descritas en el tratamiento de las hemorroides internas es importante asociar analgésicos/AINES orales (metamizol magnésico de $0,5-2 \mathrm{~g}$ o ibuprofeno de 400-600 mg cada $8 \mathrm{~h}$ ), baños de asiento con agua templada y anestésicos tópicos. Entre las primeras 24 y 72 horas debe realizarse una exéresis de la hemorroide trombosada con anestesia local. Se realiza una exéresis elíptica (en forma de ojal) eliminando la piel supralesional y el coagulo, tras infiltración de anestésico local (lidocaína al 1$2 \%$ ). Es útil añadir al anestésico local una o dos ampollas de hialuronidasa que reduce el edema instantáneamente. Si la trombosis lleva más de 
72 horas o cuando el paciente está en fase de remisión lo aconsejable es el tratamiento conservador mediante analgesia, laxantes y aconsejar dieta rica en fibra. ${ }^{(5,6)}$

\section{FISURA ANAL}

La fisura anal es probablemente la causa más común de consulta en la práctica coloproctológica. Corresponde a una solución de continuidad de la piel en el epitelio escamoso de canal anal, que se ubica en la zona distal a la línea dentada. Su localización es generalmente en la línea media posterior, y en un quinto de los casos en la línea media anterior. Las fisuras anales pueden ser agudas o crónicas, diferenciación basada tanto en el tiempo de evolución (mayor o menor de 4 a 6 semanas), como en sus características locales (profundidad, presencia de plicoma centinela, papila anal hipertrófica).

Fisiopatología: Una de las explicaciones para la fisura es la presencia de isquemia lo cal, asociada a la hipertonía anal y a una menor vascularización de los cuadrantes anterior y posterior. La etiología de la fisura anal aún no está del todo clara. Una de las explicaciones más aceptadas es que el pasaje de deposiciones voluminosas y sólidas serían factores "iniciadores" de este cuadro.

Se ha evidenciado que la mayor presión de reposo del esfínter anal, se correlaciona con menor flujo sanguíneo en la zona del anodermo, esto es más pronunciado en la zona posterior, lo cual es concordante con la distribución de la mayor parte de las fisuras; además se observa que se logra retornar a flujos considerados normales posterior a la realización de una esfiterotomía interna. Si la fisura no se ubica en la línea media $\mathrm{y} / \mathrm{o}$ es indolora, debe hacer sospechar otro tipo de patologías, como la enfermedad de Crohn, colitis ulcerosa, tuberculosis, sífilis, HIV y leucemias, entre otras. (7)

Clínica: La fisura anal se caracteriza por el intenso dolor que aparece al término de la deposición y que puede permanecer horas después de la misma. Además el paciente refiere hematoquecia y temor a la defecación. Habitualmente este episodio agudo revierte espontáneamente o con medidas higienicodietéticas produciéndose la cicatrización de la misma en 4-6 semanas. Sin embargo, en otras ocasiones el dolor y el sangrado disminuyen progresivamente sin desaparecer totalmente, presentando episodios recurrentes de reagudización y persistiendo la lesión con características crónicas a la exploración y cuyos síntomas alteran significativamente la calidad de vida del paciente. La fisura anal es la causa más frecuente de sangrado defecatorio en el niño.

Diagnóstico: El diagnóstico se establece mediante la inspección anal, puede realizarse en posición genupectoral o en decúbito lateral con flexión de caderas y rodillas. Normalmente el paciente tiene temor a ser examinado, por lo que debe procederse con cuidado. Las nalgas deben separarse suavemente y el paciente debe simular la maniobra defecatoria, así se exterioriza el anodermo y se facilita la demostración de la fisura. Habitualmente no se debe practicar tacto rectal, a no ser que la sospecha de otra patología (como un cáncer) sea alta. ${ }^{(2)}$

\section{Figura 4. Fisura anal aguda}

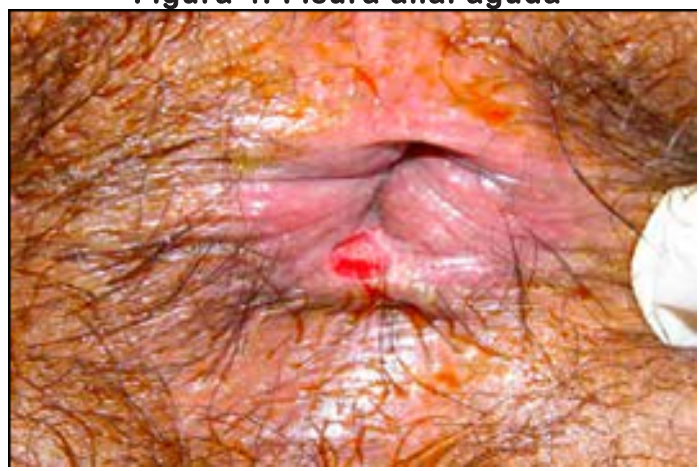

Fuente: Gastroenterology \& Hepatology Volume 10, Issue 5 May 2014

Tratamiento: casi la mitad de los pacientes con fisura anal aguda se curará con medidas de soporte, como baños de asiento, Psyllium y agentes formadores de masa fecal, con o sin la adición de anestésicos tópicos o pomadas antiinflamatorias. Se puede lograr alivio sintomático del dolor y el sangrado sin efectos adversos. La base del tratamiento es lograr disminuir la presión del esfínter anal interno, ya que esto permite que la vascularización local mejore y la cicatrización sea efectiva.

La fisura anal crónica debe tratarse con nitratos tópicos, aunque los nitrato son ligeramente superiores al placebo con respecto a la cicatrización. Los nitratos tópicos como el ungüento de nitroglicerina al $0.2 \%$ aplicado dos veces al día por 6-8 semanas se ha asociado con 
curación en al menos $50 \%$ de las fisuras crónicas y le uso de nitroglicerina tópico reduce significativamente el dolor durante el tratamiento. El principal efecto adverso es cefalea, la cual es dosis-dependiente y ocurre en $20-30 \%$ de los pacientes tratados. Los rangos de recurrencia son significativamente más altos comparados con la cirugía.

La fisura anal crónica puede también tratarse con bloqueadores de canales de calcio tópicos, con menores efectos adversos que los nitratos. La información es insuficiente para concluir si son superiores al placebo en curar la fisura anal. Los bloqueadores de canales de calcio como el diltiazem aplicado dos veces al día por 6-8 semanas se ha asociado a curación de la fisura anal en 65 a 95\% de los pacientes. Los efectos adversos incluyen cefalea, pero con menor frecuencia que con los nitratos. El diltiazem tópico puede ser el tratamiento tópico preferido por tener menor incidencia de cefalea $y$ recurrencia de la fisura anal que los nitratos. Los bloqueadores de calcio orales puede que sean igual de efectivos que los tópicos.

Figura 5. Fisura anal crónica con pliegue centinela.

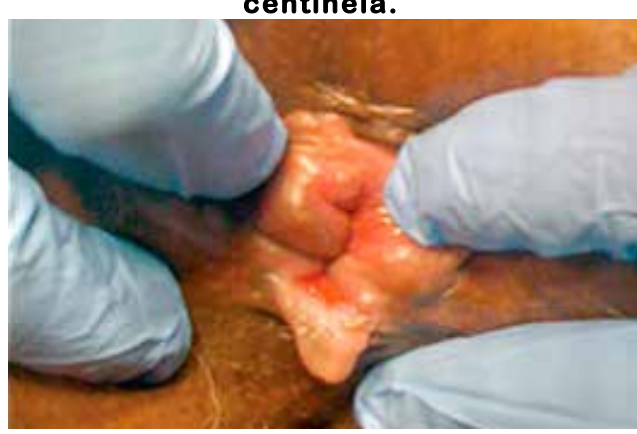

Fuente: Gastroenterology \& Hepatology Volume 10, Issue 5 May 2014

La inyección de toxina Botulínica tiene rangos de curación superiores que el placebo, aunque tiene la desventaja de requerir una inyección en un área sensible. No hay datos precisos de la dosis, sitio de aplicación, número de inyecciones o su eficacia. El efecto adverso más común es incontinencia temporal de flatos y heces. En pacientes en los que la toxina botulínica falla deben recomendarse para esfinterectomía lateral interna.

Nociones básicas del manejo quirúrgico: aquellos pacientes que son refractarios al tratamiento médico y siempre como último recurso, se contempla el tratamiento quirúrgico.
Este consiste en practicar una esfinterotomía controlada del esfínter anal interno. Este procedimiento no está exento de complicaciones, siendo el más temido la incontinencia fecal, aunque la incidencia es baja, sin embargo las tasas de curación son superiores al 90\%.

Figura 6. Algoritmo manejo de la fisura anal en atención primaria

Fuente: Manual AEC de proctología

\section{ABSCESOS Y FÍSTULAS ANORECTALES}

Un absceso anorrectal corresponde a una acumulación de material purulento en una región o espacio cercano al ano o al recto y puede drenar su contenido a través de un orificio en la piel perianal o en la mucosa rectal, pudiendo de esta manera dar origen a la formación de una fístula, la cual corresponde a un conducto de paredes fibrosas que comunica a la cripta anal que le dio origen con la piel perianal o con el recto; el orificio localizado en la cripta se denomina primario y el cutáneo o mucoso, orificio secundario. Por lo que los abscesos anorrectales y las fístulas anorrectales constituyen un padecimiento asociado $\mathrm{y}$ evolutivo, el primero como la forma aguda y el segundo como la fase crónica. 
Fisiopatología: En 1878 Chiari postuló la teoría del origen criptoglandular, la cual señala que la cripta anal sufre una infección y se obstruye. Los abscesos anorrectales se denominan primarios o inespecíficos cuando tienen un origen criptoglandular; y secundarios o específicos cuando se relacionan con otras enfermedades, como la enfermedad de Crohn, tuberculosis, traumatismos, cirugía anorrectal previa, cáncer anal o rectal, radiación, linfomas, leucemias, entre otras causas. La mayoría de los abscesos anorrectales tienen un origen criptoglandular (90 a 97\%). Las criptas anales pueden obstruirse por traumatismos, cuerpos extraños o materia fecal. La mayor incidencia de los abscesos anorrectales ocurre en la tercera y cuarta décadas de la vida. Su frecuencia es mayor en los hombres que en las mujeres (de 2:1 a 3:1). En aproximadamente $30 \%$ de los pacientes con abscesos anorrectales existe una historia previa de abscesos similares que se resolvieron espontáneamente o que requirieron intervención quirúrgica. Parece haber una mayor incidencia de abscesos y fístulas anorrectales durante las estaciones de primavera y verano.

Clasificación: De acuerdo con el espacio que afectan, los abscesos se clasifican en: interesfintéricos, perianales isquiorrectales, submucosos, pelvirrectales, o bien, puede haber afectación de más de un espacio, como ocurre en los abscesos en herradura. El tipo de absceso más frecuente es el perianal, seguido por el isquiorrectal. Los menos frecuentes son los supra-elevadores. Las fístulas se clasifican según la trayectoria que siguen a través de estos espacios y la relación que guardan con respecto a los esfínteres anales; la clasificación actualmente más utilizada es la de Parks, en la cual se consideran cuatro tipos principales de fístulas. ${ }^{(8)}$

\section{Figura 7. Clasificación Parks de las fístulas perianales}

Fuente: Rev Hosp Jua Mex 2013; 80(4): 243-247

Clínica y diagnóstico: La localización clásica de los abscesos anorectales por orden de frecuencia es el siguiente: perianal $60 \%$, isquiorrectal $20 \%$, interesfinteriano $5 \%$, y submucoso $1 \%$. La presentación clínica se correlaciona con la localización anatómica del absceso. El dolor es el síntoma principal, por lo general es muy intenso e incapacitante, de tipo punzante y que se intensifica al sentarse, deambular, toser o estornudar. Se puede presentar una zona de inflamación dolorosa, con hipertermia e hiperemia local; también se puede agregar supuración transanal, fiebre y rectorragia. El estado general no se encuentra afectado, a menos que coexistan otras enfermedades como diabetes o estados de inmunosupresión; en estos enfermos la evolución es muy rápida y grave, pudiendo extenderse en todo el periné, dando lugar a una gangrena de Fournier, que puede poner en riesgo la vida del paciente. $(2,8)$

El absceso supraelevador es diferente, pues la inspección no provee ninguna información. Los paciente frecuentemente experimentan síntomas generales como fiebre y malestar general. En estos casos, la palpación rectal revela frecuentemente induración o inclusive fluctuación. Los pacientes experimentan dolor sordo en la pelvis inferior o dolor de espalda. En los casos en los que ya se ha establecido una fístula la molestia más frecuente es la presencia de uno o varios orificios en la periferia de la apertura anal por los cuales drena secreción purulenta en forma continua o intermitente, rara vez se expulsa gas o materia fecal durante la defecación a través de estos orificios; la presencia de secreción o heces irrita la piel y ocasiona escozor y prurito. El dolor por lo general es poco intenso; sin embargo, en los casos en los que hay alguna ramificación sin drenaje libre la molestia puede ser constante y progresiva, cuando también se ocluyen los orificios secundarios perianales reaparecen síntomas y signos similares a los del absceso anorrectal. $(8,9)$

Exploración física: Para la exploración el paciente debe ser colocado en una posición que permita una inspección y una palpación adecuadas, se recomienda la posición de Sims con buena iluminación; se debe iniciar con la inspección de la región perianal, perineal, glúteos y región sacroccígea; por lo general se observa una tumefacción con hiperemia local, a la palpación puede haber hipertermia y la tumefacción puede ser fluctuante y muy dolorosa. Durante el tacto rectal se deben buscar puntos de abombamiento y dolor en el conducto anal y parte baja del recto; en ocasiones el guante sale manchado con material purulento, en algunos pacientes se puede realizar anoscopia, maniobra que permite observar la cripta afectada drenando al nivel de la línea dentada. En la actualidad el 
ultrasonido endoanal y endorrectal, la tomografía computada y la resonancia magnética, permiten evaluar la extensión y profundidad de los abscesos. ${ }^{(8)}$

\section{Figura 8. Secreción purulenta transanal en paciente con absceso rectal}

Fuente: Rev Hosp Jua Mex 2013; 80(4): 243-247

Tratamiento: Un absceso anorectal debe ser tratado mediante incisión $\mathrm{y}$ drenaje. La utilización de antibióticos sin drenaje, aunque continúa siendo una práctica demasiado habitual, no se ha demostrado útil para el tratamiento de los abscesos anorrectales y puede llevar a la progresión del proceso séptico. El drenaje se debe realizar lo más cerca del ano posible para que el trayecto de una posible fístula posterior sea más corto. Los abscesos perianales pequeños se pueden drenar en una consulta bajo anestesia local, en el centro de atención primaria, pero si hay dudas diagnósticas, signos de celulitis sin clara fluctuación, o localizaciones más profundas, es recomendable realizar la intervención en quirófano.

-Perianal: Los abscesos perianales pueden ser drenados en la consulta en muchas ocasiones. La piel es anestesiada con anestesia local y la cavidad es drenada con una incisión cruzada tan cerca al borde anal como sea posible.

-Isquiorrectal: Los abscesos isquiorrectales pequeños pueden ser drenados algunas veces en la consulta, pero los abscesos grandes son preferiblemente drenados en el quirófano

-Interesfinteriano: Los abscesos interesfinterianos deben ser drenados en el quirófano donde se puede colocar una valva en el canal anal permitiendo acceso a la cavidad a través del músculo del esfínter interno. ${ }^{(2)}$

-Abscesos más complejos, supraelevadores, postanales profundos o en herradura deben ser drenados en quirófano y bajo anestesia general.

Los antibióticos sólo están indicados en los casos en lo que la infección de tejidos blandos es extensa y abarca el periné u otras regiones más distales al sitio inicial de la infección; también se indican en pacientes con enfermedad valvular cardiaca, con prótesis óseas, diabéticos, inmunocomprometidos, y en enfermos con SIDA.
En estos casos se recomienda el empleo de antibióticos de amplio espectro tanto para aerobios como para anaerobios, y durante un lapso de siete días después del drenaje amplio del proceso infeccioso. La principal complicación del drenaje de un absceso anorrectal es la formación de una fístula, que se presenta en $60 \%$ de los casos. Los cuidados postoperatorios incluyen baños de asiento con agua tibia, laxantes formadores de bulto y analgésicos. El sangrado y drenaje suelen ceder en pocos días y las heridas deben cicatrizar en pocas semanas.

Se suele recomendar el seguimiento del paciente por parte del médico de atención primaria o cirujano para valorar una posible recurrencia del absceso (que puede ocurrir en aproximadamente $10 \%$ de los pacientes), o el posible desarrollo de una fístula posterior. El tratamiento de las fístulas anorrectales es quirúrgico y está determinado por la clasificación preoperatoria de la fístula y por la relación de su trayecto con los esfínteres del ano; los principales riesgos en el tratamiento de las fístulas anorrectales son la recurrencia de las mismas y la incontinencia fecal $^{(2,8)}$

\section{SINUS PILONIDAL}

La enfermedad pilonidal fue descrita en 1833 por Herbert Mayo y fue defiida como un quiste lleno de pelo en la región del coxis. En 1880, Hodge acuñó el término pilonidal derivado del latín pilus (pelo) y nidus (nido) para hacer referencia a su semejanza con un nido de pelos. La importancia de la enfermedad pilonidal radica en la relación tiempo/hombre perdido en sus actividades diarias secundario a sus múltiples hospitalizaciones y visitas requeridas para el manejo de la enfermedad, así como de las complicaciones asociadas a los procedimientos para tratar la enfermedad.

Fisiopatología y factores de riesgo: Existen dos teorías en cuanto a la fiiopatología de la enfermedad. La primera habla de una predisposición genética y hace mención de la probabilidad de la enfermedad como un remanente del canal medular y una consecuente invaginación del epitelio; esto fue descrito por Patey y Scarff en 1946, siendo en nuestros días una teoría en desuso. Actualmente se reconoce la enfermedad pilonidal como un padecimiento adquirido, con cierta predisposición ocupacional, ya que es muy raro en niños y se presenta más 
frecuentemente en aquellos pacientes masculinos con hirsutismo. Se han documentado múltiples factores predisponentes de esta enfermedad, sin embargo, se consideran la obesidad, el estilo de vida sedentario, un fenotipo hirsuto, una historia familiar, el trauma y la irritación local como los principales. La enfermedad pilonidal también se ha asociado a la presencia de un carcinoma en la región del seno, lo cual se reconoce en el $0.1 \%$ de los pacientes no tratados. ${ }^{(10)}$

Clínica: La presentación y diagnóstico de un seno pilonidal puede ser tan simple como un hallazgo en un paciente atendido por otra patología, con un cuadro agudo manifestado como un absceso, muy doloroso y con celulitis, o como un seno con drenaje de material purulento en forma intermitente cuando se presenta en forma crónica. La enfermedad se manifiesta como una depresión cutánea en el pliegue interglúteo (orificio primario) que se extiende mediante un tracto fibroso subcutáneo (sinus pilonidal) hasta uno o más orificios secundarios, habitualmente localizados fuera de la línea media interglútea, através de los cuales drena líquido sero-hemático o sero-purulento y, ocasionalmente, se puede objetivar la salida de pelos. $^{(2,10)}$

Tratamiento: El tratamiento médico de la enfermedad pilonidal se basa en el control local del pelo, el cual tradicionalmente se realiza con el rasurado de la zona afectada, así como en cambios en los hábitos higiénicos. Con estas dos medidas se han logrado disminuir las admisiones hospitalarias, el número de procedimientos quirúrgicos realizados y el tiempo de regreso a las actividades cotidianas. La recomendación es realizar el rasurado dos pulgadas alrededor del pliegue glúteo de la zona afectada, con borde inferior en el ano. El uso de antibióticos como terapia para esta enfermedad es limitado y aunque se reconoce que en forma preoperatoria ha logrado disminuir complicaciones de la herida, mejorar la cicatrización y disminuir la recurrencia en algunos estudios, esto no es una información concluyente. Los antibióticos se indican en aquellos pacientes inmunosuprimidos, con un área de celulitis importante o con enfermedades sistémicas concomitantes.

Una vez realizado el curetaje y la limpieza de la zona, así como de los residuos de pelo en el seno, se han descrito como tratamientos defiitivos la inyección de alcohol al $80 \%$ y el uso de pegamentos de fibrina. Si bien las diversas series que los mencionan refieren una eficacia del 60 hasta un 100\%, la realidad es que se dispone de muy poca evidencia para poder recomendar estos tratamientos de forma rutinaria.

Tratamiento quirúrgico: Cuando el paciente consulta por un absceso pilonidal se debe proceder al desbridamiento del mismo, practicando una incisión longitudinal paralela a la línea media para drenar el contenido purulento, dejando la herida abierta para que cicatrice por segunda intención (en unas 4-10 semanas). En general, este tratamiento es eficaz hasta en el $60 \%$ de los casos como procedimiento primario, sin requerir una nueva intervención. El resto de los pacientes requerirán un segundo procedimiento para tratar el seno en forma crónica, y se ha reportado que hasta el 10\% de ellos presentarán un nuevo absceso. Los mejores resultados se presentan con aquellos procedimientos en los que se realiza un colgajo con cierre fuera de la línea media, los cuales muestran un tiempo de cicatrización más rápido $\mathrm{y}$ un índice menor de recurrencia. ${ }^{(2,10)}$

Para el tratamiento de un seno pilonidal sin absceso, en forma aguda, básicamente se reconoce la resección del mismo con cierre primario de la herida, marsupialización de la misma o el manejo de la herida en forma abierta sin marsupialización. En forma general, se ha descrito una mayor tasa de cicatrización con el cierre primario de las heridas, sin embargo se ha observado un mayor índice de recurrencias con este procedimiento. De forma contraria, se presenta una cicatrización mucho más lenta en aquellas heridas manejadas en forma abierta $o$ con marsupialización, siendo el índice de recurrencia mucho menor. En estos casos, recientemente también se han utilizado los sistemas de cierre de heridas con presión negativa (VAC) para lograr una cicatrización más rápida. ${ }^{(10)}$

\section{Figura 9. A. Sinus pilonidal. B. Absceso} pilonidal agudo

Fuente: Manual AEC de proctología

En resumen, los abscesos pilonidales deben ser drenados y desbridados de forma urgente en atención primaria o en el Servicio de urgencias, tras lo cual deben ser referidos para valoración especializada en la consulta externa de cirugía. 
Los sinus pilonidales crónicos y/o complicados, deben ser derivados a la consulta externa de cirugía, para recibir tratamiento definitivo. ${ }^{(2)}$

\section{CONCLUSIÓN}

La patología anorectal incluye entidades clínicas con una elevada frecuencia de presentación en la consulta de atención primaria, aunque son para el paciente padecimientos difíciles de manifestar en la consulta. Es de considerable importancia para el médico de atención primaria conocer los aspectos básicos sobre estas entidades clínicas, su presentación, métodos diagnósticos y sobre todo su manejo y el reconocimiento del momento en que una de estas situaciones clínicas requiere manejo por parte de un médico especialista. El papel del médico no es únicamente el manejo y reconocimiento de la enfermedad sino también la prevención de la misma por lo cual es de suma importancia la búsqueda de factores de riesgo. Las patologías del ano-recto son en su mayoría capaces de resolver con un tratamiento conservador, indicado desde una consulta de primer nivel, y son, indiscutiblemente, parte de las enfermedades por las cuales el paciente más agradece al médico por su resolución.

\section{BIBLIOGRAFÍA}

1. Maksimovic J Maksimovic M. From history of proctology. Arch Oncol. 2013; 21 (1): 28-33

2. Ramos J Ciga M. Manual AEC de proctología para la atención primaria. Asociación española de Cirujanos. Madrid. Bate Scientia Salus, 2014.

3. Greenfield L Mulholland M. Greenfield's surgery. Lippincott Williams \& Wilkins. Philadelphia. 5th ed, 2011.

4. Wald A Bharucha A Cosman B Whitehead W. ACG Clinical Guideline: Management of Benign Anorectal Disorders. Am J Gastroenterol. 2014; 109(8): 1141-1157.

5. Ponce J Castells i Garangou A Gomollón F. Tratamiento de las enfermedades gastroenterológicas. Asociación Española de Gastroenterología. Barcelona. 3rd ed, 2011.
6. Sanfélix J Uribe N Campos J Reig B Bisbal E Vicedo. La patología anorrectal en la práctica clínica del médico de familia. rvmf. 2001; 05(1): 42-47

7. Wainstein C Zárate A. Urgencias proctológicas. Revista Médica Clínica Las Condes. 2011; 22(5): 677-684.

8. Rodríguez U. Abscesos y fístulas anorrectales. Rev Hosp Jua Mex. 2013;

80(4): 243-247

9. Ommer A Herold A Berg E Fürst A Sailer M Schiedeck T. German S3 guideline: anal abscess. Int J Colorectal Dis. 2012; 27:831-837

10. Salgado N Vergara O. Resultados basados en evidencia y nuevos tratamientos de la enfermedad pilonidal. Cirujano General. 2011; 33 (1): 86-89 